\title{
Factores condicionantes y dependencia espacial en el grado de concentración salarial en España*
}

\author{
BEATRIZ LARRAZ ${ }^{\text {a }}$, MARCos HerRera ${ }^{\text {b }}$ \\ a Universidad de Castilla-La Mancha, Facultad de Ciencias Jurídicas y Sociales, c/ Cobertizo de San \\ Pedro Mártir, s/n, 45071 Toledo, España.E-mail: beatriz.larraz@uclm.es \\ b CONICET-IELDE, Universidad Nacional de Salta, Avda. Bolivia, 5150 (A4408FVY), Salta, \\ Argentina.E-mail: mherreragomez@conicet.gov.ar
}

\begin{abstract}
RESUMEN
En los últimos años, el desigual reparto salarial en España parece preocupar cada vez más. Tratando de contribuir a la literatura existente hasta el momento, en este artículo se analizará el impacto que tienen las características personales y laborales del trabajador y las de la empresa en la distribución de los salarios. El análisis se ha llevado a cabo a partir de los últimos micro-datos oficiales disponibles sobre salarios, correspondientes a la Encuesta de Estructura Salarial del año 2010 en las 52 provincias españolas. Utilizando los últimos desarrollos de datos de paneles espaciales, se analiza la posible dependencia espacial y las diferentes alternativas de especificación. Los resultados muestran que el modelo $S L X$, con efectos fijos, es adecuado para explicar la desigualdad salarial.
\end{abstract}

Palabras clave: Concentración salarial, desigualdad, Gini, modelos econométricos espaciales.

\section{Determinants of Wage Inequality in Spain: A Spatial Approach}

\begin{abstract}
In recent years, unequal wage distribution in Spain appears to be of particular importance. This article seeks to contribute to the literature so far going deeper into the knowledge of the possible determinants of this wage concentration in Spain. The impact of workers' personal and employment characteristics and those of the company in the distribution of wages will be analyzed. The analysis was carried out from the latest available official micro-data on wages corresponding to the Wage Structure Survey 2010 in 52 Spanish provinces. Subsequently, using the latest developments in spatial panel data, any possible spatial dependence and the different specification options will be analyzed. The results show that fixed-effect $S L X$ is appropriate to explain wage inequality. The sampling strategy means that the final model must be includes a correction of nuisance parameters due to the correlation between consecutive samples.
\end{abstract}

Keywords: Wage Inequality, Gini Index, Spatial Econometric Models.

Clasificación JEL: J01, J16, J31, O15, C18

\footnotetext{
* Los autores agradecen el apoyo financiero recibido para llevar a cabo la investigación y la publicación de este artículo a la Subvención del Ministerio de Economía y Competitividad CSO2013-43054-R (Beatriz Larraz) y ECO2012-36032-C03-01 (Marcos Herrera).
}

Artículo recibido en marzo de 2016 y aceptado en mayo de 2016

Artículo disponible en versión electrónica en la página www.revista-eea.net, ref. ə-34301 


\section{INTRODUCCIÓN}

Es un hecho indudable la creciente preocupación que existe por el incremento en la desigualdad de renta disponible en los hogares en las últimas décadas (Stiglitz, 2012; Milanovic, 2012) y las implicaciones que este hecho tienen en la sociedad. Según Vicente-Perdiz et al. (2010), todo parece indicar que la desigualdad mundial es inquietante, debido a su espectacular aumento en el largo plazo. Entre otros problemas derivados, parece que crecientes desigualdades en la renta hacen que descienda la confianza en gobiernos e instituciones, como es el caso del estudio llevado a cabo en países latinoamericanos (Zmerli y Castillo, 2015) o incluso que disminuya el bienestar infantil, según el estudio realizado en un conjunto de países europeos (Pickett y Wilkinson, 2015), mientras que hasta pueden verse incrementados los problemas de salud, como es el caso del estudio sobre la población en Estados Unidos (Pabayo et al., 2015), por poner algunos ejemplos. Otros autores, como Castells-Quintana y Royuela (2014) afirman que la presencia de altos niveles de desigualdad puede ser un factor limitante incluso para el crecimiento a largo plazo del país.

En el caso de España, según el informe del Fondo Monetario Internacional (FMI, 2012), el índice de Gini (Gini, 1914) sobre la renta disponible ha avanzado casi tres puntos porcentuales, desde 31,8 en 1980 a 34,7 en 2010, mientras que en otras economías avanzadas como el Reino Unido o Estados Unidos, los incrementos han sido incluso de 6,5 y 7,5 puntos porcentuales, respectivamente, habiendo pasado de 27,0 a 33,5 en el caso del Reino Unido y de 30,1 en 1980, a un más que preocupante 38,6 en EEUU en el mismo periodo. En el caso de China, el último dato disponible de 2005 sitúa su índice Gini de desigualdad en 41,5, y para Latinoamérica y la zona del Caribe este coeficiente incluso alcanza los 47,7 puntos sobre 100 .

Algunos autores han tratado de identificar los posibles motivos de dichos incrementos de desigualdad. Por ejemplo, Piketty (2013) sostiene que dado que el rendimiento del capital ha sido mayor al crecimiento de la economía a escala mundial, fueron quienes contaban inicialmente con ese capital los que lograron mayor beneficio de dicho crecimiento que quienes dependían de su trabajo, incrementándose así las diferencias entre unos y otros. En concreto, en España, el periodo 1998-2005 fue un periodo de crecimiento económico pero con escasa reducción de la desigualdad (Ochando, 2010) debido, entre otros motivos, a los cambios acaecidos en el mercado de trabajo, que experimentó creación de empleo, pero de baja productividad y bajos salarios y con un aumento de la desigualdad salarial. Hassine (2015) señala a la composición demográfica, el capital humano y las características concretas de la comunidad como las principales fuentes de las diferencias de dotaciones en los hogares urbanos frente a los rurales en el caso concreto de los 12 países árabes estudiados. Mientras que entre regiones metropolitanas y no metropolitanas, la desigualdad vendría fun- 
damentalmente dada por las diferencias en los rendimientos del capital humano. En el caso de Estados Unidos, Gilens (2012) llega a afirmar en su estudio que los resultados de las políticas están más fuertemente correlacionados con las preferencias de los ricos que con las de los pobres, siendo el incremento de la desigualdad una clara consecuencia de este hecho. Por su parte, Kristal y Cohen (2015) afirman que el declive de los sindicatos unido a la caída del valor real del salario mínimo explican la mitad de la creciente desigualdad, mientras que la tan citada informatización y sociedad de la tecnología son capaces de explicar sólo la quinta parte. Sugieren que mucha de la responsabilidad de la desigualdad viene motivada por la falta de empoderamiento de los trabajadores más que por las fuerzas del mercado. Por su parte, Castells-Quintana et al. (2015) afirman que la especialización del sector terciario y el cambio tecnológico son factores asociados con el aumento de las desigualdades.

Como posibles soluciones a esta realidad, Hatch y Rigby (2015) afirman que la desigualdad puede verse reducida con una combinación de altos impuestos a las clases altas, bajos impuestos a los más pobres y regulaciones del mercado laboral que sean favorables a los trabajadores, como podría ser la existencia de un salario mínimo. Así mismo, Park y Mercado (2015) concluyen del estudio llevado a cabo en economías de Asia y el Pacífico que la inclusión financiera puede reducir la pobreza y la desigualdad económica de una sociedad.

Además de los diversos motivos del incremento de la desigualdad apuntados, siguiendo a Ochando (2010), en nuestra opinión, esta desigualdad en la renta disponible tiene su origen, en parte, en la desigual distribución de los salarios entre los trabajadores asalariados, ya que, en general, altos salarios implican alta renta disponible y viceversa. De hecho, en opinión de Palacios et al. (2014), es este aumento en la polarización salarial el que está acentuando las tensiones sociales y, por tanto, debe tenerse en cuenta en el diseño de políticas económicas y sociales. Desde este punto de vista, el estudio de la desigualdad en el reparto de la masa salarial entre la población de un país resulta especialmente relevante. Tratando, por tanto, de contribuir a la literatura sobre desigualdad económica, sus causas y posibles soluciones, en este artículo se ha profundizado en los posibles determinantes de la desigual distribución salarial en España. Se analizará el impacto que sobre dicha distribución salarial tiene el nivel educativo alcanzado por el trabajador, el tipo de ocupación que desempeña en el puesto de trabajo, el sector económico en el que se encuadra la actividad de la empresa o la experiencia acumulada por el mismo, entre otras. El análisis se ha llevado a cabo a partir de los últimos datos oficiales disponibles sobre salarios, correspondientes a la Encuesta de Estructura Salarial del año 2010 (EES, 2012a) en las 52 provincias españolas.

Además, el trabajo presenta evidencia de existencia de correlación espacial positiva entre regiones geográficamente adyacentes, incorporando al análisis el 
impacto del entorno socioeconómico de las regiones vecinas. La lectura conjunta de determinantes propios, como el análisis del vecindario, genera una mejor comprensión de los factores que impactan sobre la concentración salarial. Dichos factores serán estimados siguiendo el modelo econométrico con efectos espaciales existente más adecuado (véase una aplicación en Maté et al., 2009) considerando efectos fijos e incorporando el modelo más reciente de Halleck Vega y Elhorst (2015).

El resto del artículo se organiza como sigue. En la Sección 2 se describen los datos utilizados en el estudio así como el método empleado para medir la concentración salarial. En la Sección 3 se lleva a cabo el análisis espacial de dicha concentración salarial en España, cuya consideración es fundamental para la decisión del modelo econométrico a utilizar. La Sección 4 detalla el modelo que analiza la influencia en la desigualdad en la distribución salarial de algunos determinantes potenciales de efectos fijos para datos espaciales. En la Sección 5 se muestran los resultados, finalizando con las conclusiones del estudio en la Sección 6.

\section{DATOS Y MÉTODO PARA MEDIR LA DESIGUALDAD EN LA DISTRIBUCIÓN DE LOS SALARIOS}

Los datos utilizados para el presente estudio de la desigualdad salarial provienen de la última información disponible con micro-datos sobre la distribución de los salarios en España, incluida en la Encuesta de Estructura Salarial de 2010 (EES, 2012a), de carácter cuatrienal. A través de dichos micro-datos se puede disponer de los salarios individuales de los trabajadores en función de sus características personales, laborales y del centro en el que desempeñan su labor (Tabla 1), así como de la provincia en la que desempeñan su puesto de trabajo. La fuente primaria de los datos proviene de los centros de cotización seleccionados en la muestra, excluyendo las empresas del sector agrícola y pesquero, trabajadores de la administración pública no incluidos en la Seguridad Social, personal doméstico y trabajadores de organismos extraterritoriales.

En este estudio se ha tenido en cuenta la variable "ganancia bruta anual por trabajador", que incluye pagos en especie, con el fin de estudiar los diferentes grados de concentración existentes en los salarios brutos anuales. Dado que la presencia de un mayor grado de desigualdad en el salario bruto anual percibido por los trabajadores redunda en una creciente disparidad de rentas disponibles, a través del grado de concentración de dicha variable podrán estudiarse los determinantes de las crecientes desigualdades salariales en España, es decir, hasta qué punto éstos son consecuencia de las características personales o laborales del trabajador, de las características de la empresa o, incluso, de la dimensión geográfica. 


\section{Tabla 1}

Descripción de las variables y los grupos considerados en el estudio

\begin{tabular}{ll}
\hline \multicolumn{1}{c}{ Variable } & \multicolumn{1}{c}{ Grupos } \\
\hline Edad & 16-25 años; 25-35 años; 35-55 años; >55 años \\
Nacionalidad & Española; resto del mundo \\
Nivel de estudios & Sin estudios; Primarios; Secundarios; FP1; FP2; Universitarios; \\
Años de antigüedad & <2 años; de 2 a 10 años; > 10 años \\
Tipo de jornada & Completa; parcial \\
Tipo de contrato & Indefinido; temporal \\
Responsabilidad del trabajador & Responsable; No responsable \\
Ocupación del trabajador & Clasificación Nacional de Ocupaciones (CNO, 2011) \\
Actividad de la empresa & Clasificación Nacional de Actividades Económicas (CNAE, 2009) \\
Tamaño de la empresa & Micro empresa; pequeña; mediana; grande \\
Control & Público; privado \\
Tipo de mercado principal & Local o regional; nacional; internacional \\
Convenio colectivo & Superior a la empresa; de empresa (o inferior) \\
\hline
\end{tabular}

Fuente: Elaboración propia a partir de la metodología de la EES (2012b).

La encuesta llevada a cabo, fechada el mes de octubre de 2010, recoge información acerca de 25.104 cuentas de cotización de la Seguridad Social y de 216.769 trabajadores de las mismas, correspondiendo el factor de elevación al número de trabajadores de la población española que se corresponden con dicha información.

Para realizar la medición cuantitativa del grado de concentración, el índice de Gini (Gini, 1914) continua constituyendo el coeficiente más utilizado por las agencias de estadística oficial (EES, 2012b, Eurostat, 2012) y también en la literatura científica (Giorgi, 2005; Basulto y Busto, 2010). En el caso del estudio de ingresos o salarios, dicho índice de concentración está basado en la relación entre la proporción acumulada de población $p_{i}=i / n$, siendo $n$ el número de individuos, y la de ingresos $q_{i}=A_{i} / A_{n}$, donde $A_{i}=\sum_{k=1}^{i} x_{k}$ y $\left\{x_{i}\right\}_{i=1}^{n}$ representan los ingresos individuales ordenados de menor a mayor:

$$
I G=\frac{\sum_{i=1}^{n-1}\left(p_{i}-q_{i}\right)}{\sum_{i=1}^{n-1} p_{i}}
$$

Los valores de dicho índice oscilan entre el cero, que se corresponde con un nivel de equidistribución total, y uno, de máxima concentración económica o desigualdad total en el reparto de la variable. Una expresión equivalente, que devuelve exactamente el mismo resultado (Larraz, 2015), es la basada en la 
definición de diferencia media de Gini (Gini, 1912) y que viene dada por la expresión:

$$
I G=\frac{\sum_{i=1}^{n} \sum_{j=1}^{n}\left|x_{i}-x_{j}\right|}{2 \bar{x} n(n-1)}
$$

siendo $\bar{X}$ la media aritmética de los ingresos.

A pesar de la vigencia de estas definiciones, cabe resaltar el hecho de que la expresión (1) sólo puede ser aplicada en caso de que las frecuencias sean unitarias, por lo que su uso con datos de encuesta está limitado, ya que el factor de elevación implica la repetición de cada ingreso un número de veces, no entero en la mayor parte de las ocasiones. Para superar esta limitación, cuando las frecuencias disponibles son no unitarias, el cálculo del índice de desigualdad de Gini puede realizarse a través de la siguiente expresión (3), siendo $n_{i}$ el factor de elevación correspondiente al individuo $i$ y $N$ el número total de trabajadores en la población española:

$$
I G=\frac{\sum_{i=1}^{n} \sum_{j=1}^{n}\left|x_{i}-x_{j}\right| n_{i} n_{j}}{2 \bar{x} N(N-1)}
$$

Es importante destacar que el índice de concentración utilizado no es afectado por los cambios de escala. Esto implica, por ejemplo, ante una desigualdad menor (mayor) observada en una provincia respecto a otra no respondería a una situación relativa de salarios medios menores (mayores) en la primera provincia respecto a la segunda, si no, a una realidad de menor desigualdad relativa en sí misma. Además, cabe recordar que todos los índices definidos tienen su campo de variación entre 0 y 1 , indicando a mayor valor del índice, mayor desigualdad, $\mathrm{y}$ a menor, mayor equidad.

\section{ANÁLISIS ESPACIAL AGREGADO DE LA CONCENTRACIÓN SALARIAL EN ESPAÑA}

A partir de los microdatos de la EES de 2010 (2012a) se ha calculado el índice de concentración global tal como se describe en la Sección 2 a escala provincial para España. La estrategia consistió en obtener cien muestras para cada provincia y calcular el índice de concentración de la ecuación (3) para cada una de ellas. Con ello se pretende comparar la situación de cada provincia respecto de las demás en lo referente a la desigualdad de la masa salarial anual.

Utilizando el valor promedio de todas las muestras, se obtuvo la distribución representada por el mapa de la Figura 1. La escala de grises del mapa ha sido confeccionada a partir de la distribución por cuantiles de la distribución del 
índice de concentración, expresado en términos porcentuales. El valor promedio para el agregado anual es de $31,50 \%$ con un valor mínimo de $28,18 \%$ y un valor máximo 34,46.

Figura 1

Mapa de España a escala provincial correspondiente al índice de concentración del salario anual calculado a partir del promedio de cien muestras para cada provincia

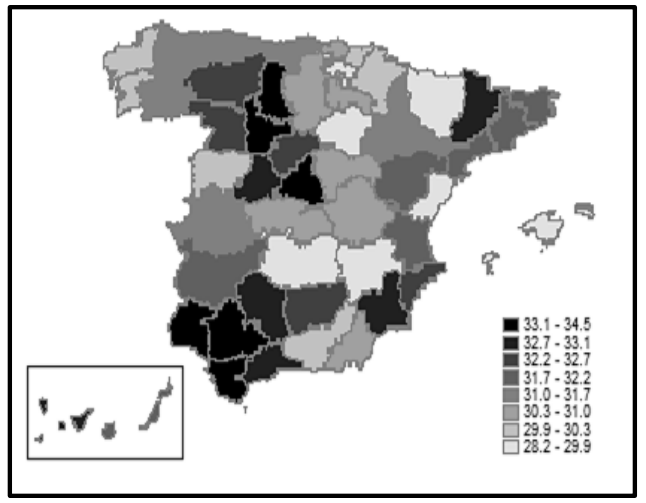

Fuente: Elaboración propia a partir de datos de EES (2012a).

Si bien dicho mapa muestra comportamientos en la variable de la desigualdad salarial que sugiere la presencia de autocorrelación espacial de la variable, resulta imprescindible llevar a cabo un análisis más detallado a través de los contrastes de autocorrelacción existentes en la literatura. En nuestro caso utilizaremos el estadístico I de Moran (1950), capaz de contrastar si se cumple la hipótesis de que los valores obtenidos del muestreo de una variable aleatoria se encuentran distribuidos de forma totalmente aleatoria en el espacio, o si por el contrario, existe una asociación significativa de valores entre regiones vecinas. En su construcción, es necesario recurrir a la denominada matriz de pesos espaciales en la que se plasmen las relaciones espaciales entre cada par de localizaciones, con el fin de definir el concepto de cercanía. En este caso concreto, se ha recurrido al concepto de contigüidad física de primer orden utilizado por Moran (1948) y Geary (1954), donde $w_{i j}$ es unitario si las regiones $i$ y $j$ son físicamente adyacentes y nulo en caso contrario. El estadístico basado en este principio fue desarrollado por Moran y se define como:

$$
I=\frac{R \sum_{i=1}^{R} \sum_{j=1}^{R} w_{i j}\left(x_{i}-\bar{x}\right)\left(x_{j}-\bar{x}\right)}{S_{0} \sum_{i=1}^{R}\left(x_{i}-\bar{x}\right)^{2}}
$$


siendo $R=52$, el número de provincias españolas y $S_{0}=\sum_{i=1}^{R} \sum_{j=1}^{R} w_{i j}$. La distribución asintótica bajo hipótesis nula del estadístico se aproxima a una normal, aunque es habitual utilizar una distribución empírica por permutación.

A través de la metodología expuesta, se presenta en la Figura 2 el diagrama de dispersión de Moran, en donde la pendiente de la línea trazada es el valor del contraste y su $p$-valor ha sido obtenido por permutación. El valor del estadístico es significativamente positivo para el salario bruto anual, por lo que se rechaza la hipótesis de ausencia de autocorrelación espacial.

Figura 2

Diagrama de dispersión de Moran correspondiente al índice de concentración del conjunto de la población calculado a partir del promedio de cien muestras de salario anual percibido por cada trabajador para cada provincia

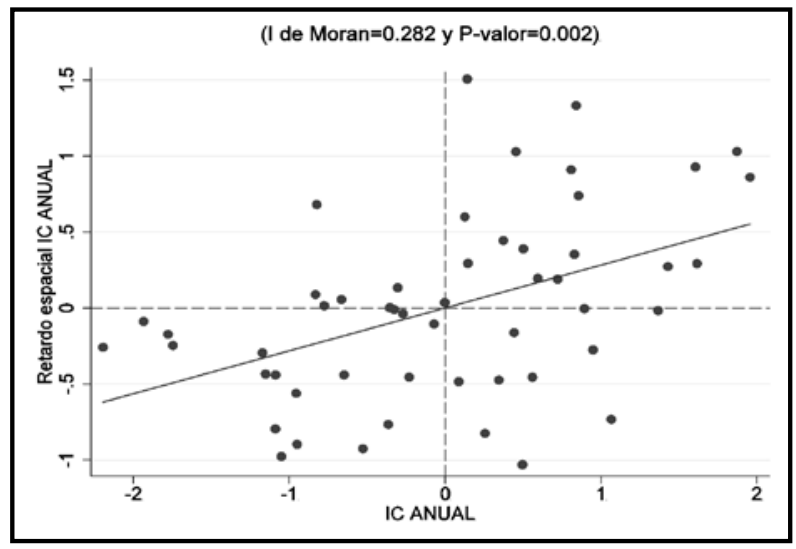

Fuente: Elaboración propia a partir de datos de EES (2012a).

\section{MODELO DE EFECTOS FIJOS PARA DATOS ESPACIALES}

Dada la evidencia a favor de correlación espacial positiva en el fenómeno de la concentración, parece clara la necesidad de especificar un modelo econométrico con efectos espaciales en el que se analice la influencia en la desigualdad en la distribución salarial de algunos determinantes potenciales (estructura demográfica, estructura educativa, estructura del mercado de trabajo, estructura sectorial, etc.). El problema para ajustar un modelo econométrico es que no se dispone de suficiente variabilidad y grados de libertad en los datos, ya que la muestra inicial de 216.769 trabajadores se ha reducido, al calcular los índices de desigualdad provinciales, a sólo 52 observaciones (una por provincia, más Ceuta y Melilla). A fin de superar esta limitación -y considerando que una selección muestral diferente hubiera dado lugar a valores, de la variable endógena (índices de Gini) y variables explicativas, diferentes- a partir de la muestra 
inicial de 216.769 trabajadores se ha generado por remuestreo (replicando el proceso muestral empleado en la selección de la muestra) 100 nuevas muestras de trabajadores (del mismo tamaño), de donde se han obtenido, para cada unidad espacial, 100 valores diferentes de índices de desigualdad y de factores explicativos potenciales. Esto permite incrementar significativamente el tamaño de la muestra con el único coste de introducir una estructura de anidamiento (multinivel) en la base de datos.

Esta sección presenta el desarrollo metodológico aplicado a la estructura de nuestros datos empíricos. La cantidad de observaciones totales son 5.200 distribuidas en 100 muestras por cada una de las 52 jurisdicciones (50 provincias más las ciudades autónomas de Ceuta y Melilla). Formalmente, un modelo de $R$ unidades regionales y $J$ muestras, puede expresarse como:

$$
Y_{j}=X_{j} \beta+\varepsilon_{j}, \varepsilon_{j} \sim N\left(0_{R}, \sigma^{2} I_{R}\right), \forall j=1, \ldots, J
$$

donde $Y_{j}$ representa la variable dependiente, siendo un vector de orden $R \times 1$, con el índice $j$ identificando a la muestra $j$-ésima; $X_{j}$ es una matriz de $R \times k$ variables explicativas; además, $\beta$ un vector de parámetros desconocidos de orden $k \times 1, \mathrm{y} \varepsilon_{j}$ es un término de error con las características habituales, $\varepsilon_{j} \sim N\left(0_{R}, \sigma^{2} I_{R}\right)$. Este modelo es conocido como pooled.

La detección de dependencia espacial en un modelo tipo pooled ha sido desarrollada por Anselin et al. (2006) mediante un conjunto de contrastes de Multiplicadores de Lagrange ( $L M)$. Los estadísticos $L M$ suponen como hipótesis nula al modelo (5) y plantean una estructura espacial bajo hipótesis alternativa $\left(H_{1}\right)$. Por ejemplo, si $H_{1}$ especifica un modelo de retardo espacial endógeno $(S L M)$ :

$$
Y_{j}=\rho W Y_{j}+X_{j} \beta+\varepsilon_{j}
$$

entonces, el estadístico es:

$$
L M_{\rho}=\frac{\left[e^{\prime}\left(I_{J} \otimes W\right) Y / \hat{\sigma}^{2}\right]^{2}}{G} \sim \chi_{1}^{2}
$$

donde $e$ representa al vector de residuos del modelo (5), con:

$$
\begin{gathered}
G=\frac{1}{\hat{\sigma}^{2}}\left[\left(\left(I_{J} \otimes W\right) X \hat{\beta}\right)^{\prime}\left(I_{R J}-X\left(X^{\prime} X\right)^{-1} X^{\prime}\right)\left(I_{J} \otimes W\right) X \hat{\beta}+J J_{W} \hat{\sigma}^{2}\right] \\
J_{W}=\operatorname{tr}\left(W W+W^{\prime} W\right)
\end{gathered}
$$

Si bajo la hipótesis alternativa se define un modelo de error espacial (SEM): 


$$
\begin{gathered}
Y_{j}=X_{j} \beta+u_{j} \\
u_{j}=\lambda W u_{j}+\varepsilon_{j}
\end{gathered}
$$

entonces, el estadístico para su detección se define como:

$$
L M_{\lambda}=\frac{\left[e^{\prime}\left(I_{J} \otimes W\right) e / \hat{\sigma}^{2}\right]^{2}}{J \times J_{W}} \sim \chi_{1}^{2}
$$

Ambos contrastes se distribuyen asintóticamente como una Chi-cuadrado con un grado de libertad. Estos contrastes $L M$ son de tipo simple y poseen la desventaja que son sensibles a la presencia de otros tipos de estructura espacial. Para evitar estos falsos positivos, se han desarrollado versiones robustas de ambos estadísticos $L M$ (véase Elhorst, 2010).

$$
\begin{gathered}
L M_{\rho}^{*}=\frac{\left[e^{\prime}\left(I_{J} \otimes W\right) Y / \hat{\sigma}^{2}-e^{\prime}\left(I_{J} \otimes W\right) e / \hat{\sigma}^{2}\right]^{2}}{G-J \times J_{W}} \sim \chi_{1}^{2} \\
L M_{\lambda}^{*}=\frac{\left[e^{\prime}\left(I_{J} \otimes W\right) e / \hat{\sigma}^{2}-J \times J_{W} / G \times e^{\prime}\left(I_{J} \otimes W\right) Y / \hat{\sigma}^{2}\right]}{J \times J_{W}\left[1-J \times J_{W} / G\right]} \sim \chi_{1}^{2}
\end{gathered}
$$

La detección de autocorrelación espacial mediante los tests $L M$ supone que los datos provienen de una muestra aleatoria. Sin embargo, por construcción, nuestros datos poseen una estructura de tipo anidada, similar a una estructura jerárquica de dos niveles (Snijders y Bosker, 1999) o a un dato de panel estático (Hsiao, 2003). Esto implica que las muestras no son independientes y el modelo debe reconocer esta situación. La forma más usual para ello es la introducción de un efecto específico para cada unidad espacial:

$$
Y_{j}=X_{j} \beta+\alpha+\varepsilon_{j}, \forall j=1, \ldots, J
$$

donde $\alpha=\left(\alpha_{1}, \ldots, \alpha_{R}\right)^{\prime}$ es un vector de efectos específicos para cada unidad espacial o jurisdicción $i$-ésima, de naturaleza fija o aleatoria para todas las $J$ muestras. En el caso de los efectos fijos, una variable ficticia es añadida por cada unidad espacial (excepto una para evitar multicolinealidad perfecta), mientras que en el caso de efectos aleatorios, los coeficientes $\alpha_{i}$ son considerados como variables aleatorias i.i.d. con media cero y varianza constante, $\sigma_{\alpha}^{2}$.

La elección entre efectos específicos fijos o aleatorios puede realizarse por medio del contraste de Hausman (1978). Bajo los supuestos habituales del modelo lineal general, los efectos aleatorios son eficientes. Sin embargo, la viola- 
ción del supuesto de exogeneidad genera inconsistencia de dichos efectos, no así de los efectos fijos. La elección entre estos efectos puede basarse en cuestiones empíricas. Por ejemplo, si la estructura de la muestra analizada posee un número de grupos relativamente pequeño con suficiente información dentro de cada grupo puede ser conveniente utilizar efectos fijos ya que requiere supuestos menos restrictivos respecto al comportamiento de los parámetros $\alpha$ 's. En el caso de una estructura panel con numerosos grupos, los efectos fijos necesitan estimar una enorme cantidad de coeficientes ocasionando una gran pérdida de grados de libertad. En este último caso, los efectos aleatorios son útiles ya que solo requieren la estimación de un único parámetro, la varianza inter-grupos, para describir la heterogeneidad de los datos. Otra diferencia entre efectos fijos y aleatorios es de tipo inferencial: los efectos fijos sólo permiten realizar inferencia respecto a la muestra analizada, mientras que los efectos aleatorios generan inferencia a nivel poblacional. Sin embargo, bajo datos espaciales, diferentes autores argumentan que la estimación por efectos fijos puede ser interpretada como de tipo poblacional si los datos espaciales cubren completamente el espacio geográfico ${ }^{1}$. Por lo tanto, dadas las características de nuestros datos con un número reducido de grupos espaciales (52 jurisdicciones) y completa cobertura del territorio español, la introducción de efectos específicos será realizada por medio de efectos fijos.

Los estadísticos $L M$ han sido extendidos para el modelo de efectos fijos, ecuación (12), y su derivación matemática puede consultarse en Debarsy y Ertur (2010). El rechazo de uno o ambos tests $L M$ conlleva a la incorporación de elementos espaciales en la especificación econométrica. Por lo general, la metodología tradicional va desde la estimación de un pooled hacia un modelo espacial SEM o SLM, estimado por métodos más complejos como (Máxima Verosimilitud) $M V$, (Método de los Momentos Generalizado) GMM o QuasiMV. Sin embargo, recientemente, Halleck Vega y Elhorst (2015) llaman la atención sobre la necesidad de realizar un paso intermedio entre el modelo pooled y los modelos con efectos espaciales, SEM o SLM. Este modelo intermedio es una extensión espacial simple conocida como modelo espacial en $X$ 's (SLX, spatial lag in $X$ 's) que posee la siguiente especificación:

$$
Y_{j}=X_{j} \beta+W X_{j} \gamma+\alpha+u_{j}
$$

donde $\gamma$ captura el impacto promedio local de las variables explicativas de los vecinos a cada unidad espacial.

La ventaja del $S L X$ respecto a otros modelos espaciales es que no es necesario recurrir a otras alternativas de estimación que las ya habituales en datos de

\footnotetext{
${ }^{1}$ La discusión entre la conveniencia entre efectos fijos y aleatorios para datos de naturaleza espacial es un tema de actual debate y no se encuentra cerrado. Para mayor detalle véase a Elhorst (2014, pp. 53).
} 
panel ( $W X_{j}$ son exógenas igual que las $X_{j}$ ). Además, la interpretación de los coeficientes es directa, en contraposición a lo que sucede para modelos con un retardo espacial endógeno (LeSage y Pace, 2009, pp. 34).

La aplicación de los contrastes $L M$, considerando al modelo (13) como hipótesis nula, genera que bajo hipótesis alternativa surjan dos modelos espaciales más complejos. Para el $L M_{\rho}$, el modelo bajo hipótesis alternativa es un (Spatial Durbin Model) SDM de efectos fijos:

$$
Y_{j}=\rho W Y_{j}+X_{j} \beta+W X_{j} \gamma+\alpha+\varepsilon_{j}
$$

Para el $L M_{\lambda}$, el modelo bajo hipótesis alternativa es un (Spatial Durbin Error Model) SDEM de efectos fijos:

$$
\begin{gathered}
Y_{j}=X_{j} \beta+W X_{j} \gamma+\alpha+u_{j} \\
u_{j}=\lambda W u_{j}+\varepsilon_{j}
\end{gathered}
$$

A continuación se utilizará lo expuesto en esta sección para detectar la especificación más adecuada para nuestros datos.

\section{RESULTADOS}

Siguiendo lo manifiesto en la Sección 2, a partir de la información de la última Encuesta de Estructura Salarial han sido calculados los índices de concentración salarial para cada provincia española. Considerando dicha información como variable dependiente, y tomando como base los modelos econométricos espaciales de la Sección 3, en esta sección se presentan las estimaciones de las variaciones del grado de concentración provincial en función de los cambios en la estructura del conjunto de trabajadores de una provincia. Así, a través de este análisis, responderemos a preguntas como ¿experimenta cambios la desigualdad salarial en función de la estructura sectorial de los ocupados de la provincia?; ¿se incrementa el grado de concentración salarial con la edad de los trabajadores?; ¿y con el nivel educativo alcanzado o el tipo de contratación?

Para llevar a cabo este objetivo, se ha planteado el siguiente modelo lineal: Concetración salarial = $f$ (características personales; laborales; de la empresa; geográficas)

El estudio ha sido llevado a cabo considerando como variable dependiente la concentración salarial medida a través del salario anual de los trabajadores (IG SAL.ANUAL). Por otro lado, la Tabla 2 muestra las variables explicativas que han sido consideradas en el modelo obtenidas a partir de la información disponible en la encuesta y consideradas en porcentaje sobre el total de los trabajadores.

De la Tabla 2 se puede deducir que se considera como clase equivalente a trabajadores entre 30 y 49 años, con estudios de grado medio, de nacionalidad 
española, con una antigüedad entre 2 y 10 años en la empresa, con contrato indefinido y a jornada completa, ocupando un puesto medio, que trabaja en una empresa dedicada al sector servicios, de menos de 200 trabajadores, del sector privado, que no exporta al exterior su producción y regida por un convenio colectivo superior al de empresa, provincial o sectorial.

Tabla 2

Variables consideradas en el modelo

\begin{tabular}{ll}
\hline Variables $^{\circ}$ & \multicolumn{1}{c}{ Definición } \\
\hline Dependiente & \\
\hline IG SAL.ANUAL & Índice de Gini provincial a partir del salario anual \\
\hline Independientes & \\
\hline EDAD 16-29 & Porcentaje de trabajadores jóvenes, entre 16 y 29 años \\
EDAD $>49$ & Porcentaje de trabajadores mayores de 49 años \\
EST. PRIMARIOS & Porcentaje de trabajadores con estudios primarios \\
EST. SUPERIORES & Porcentaje de trabajadores con estudios superiores \\
NAC. NO ESP & Porcentaje de trabajadores con nacionalidad distinta de española \\
ANTIG $<2$ & Porcentaje de trabajadores con menos de 2 años de antigüedad \\
ANTIG $>10$ & Porcentaje de trabajadores con más de 10 años de antigüedad \\
TEMPORAL & Porcentaje de trabajadores temporales \\
PARCIAL & Porcentaje de trabajadores a jornada parcial \\
OCUP.ELEMENTAL & Porcentaje de trabajadores en ocupaciones elementales \\
OCUP.SUPERIOR & Porcentaje de trabajadores en ocupaciones superiores \\
CONSTRUCCCION & Porcentaje de trabajadores en el sector de la construcción \\
INDUSTRIA & Porcentaje de trabajadores en el sector industrial \\
GRANDE & Porcentaje de trabajadores en empresas >200 trabajadores \\
PÚBLICO & Porcentaje de trabajadores en empresas con control público \\
EXTERIOR & Porcentaje de trabajadores en empresas dedicadas a la exportación \\
REG.EMPRESA & Porcentaje de trabajadores en empresas con convenio colectivo de \\
\hline
\end{tabular}

Fuente: Elaboración propia a partir de la información de los micro-datos (EES 2012a).

El primer modelo estimado es un modelo pooled (ecuación 5), cuyos resultados son resumidos en la Tabla 3. Debido a que este modelo es el más simple de los presentados, la significancia estadística no será interpretada directamente hasta tanto no se comprueben las diferentes hipótesis de independencia espacial sobre los residuos.

Tabla 3

Resultados del modelo lineal estimado por mínimos cuadrados ordinarios

\begin{tabular}{l|ccl}
\hline \multirow{2}{*}{\multicolumn{1}{c|}{ Variable }} & \multicolumn{3}{c}{ IG SAL.ANUAL } \\
\cline { 2 - 4 } & Coef. & t-test & p-valor \\
\hline Constante & 0,28596 & 44,76 & 0,000 \\
EDAD 16-29 & $-0,00073$ & $-8,34$ & 0,000 \\
EDAD >49 & 0,00022 & 2,87 & 0,004 \\
EST. PRIMARIOS & 0,00064 & 12,22 & 0,000 \\
EST. SUPERIORES & $-0,00013$ & $-1,77$ & 0,075 \\
\hline
\end{tabular}


Tabla 3 (continuación)

Resultados del modelo lineal estimado por mínimos cuadrados ordinarios

\begin{tabular}{l|ccc}
\hline \multirow{2}{*}{ Variable } & \multicolumn{3}{c}{ IG SAL.ANUAL } \\
\cline { 2 - 4 } & Coef. & t-test & p-valor \\
\hline NAC. NO ESP & 0,00017 & 2,87 & 0,004 \\
ANTIG $<2$ & $-0,00073$ & $-9,39$ & 0,000 \\
ANTIG $>10$ & $-0,00008$ & $-1,09$ & 0,277 \\
TEMPORAL & $-0,00020$ & $-2,90$ & 0,003 \\
PARCIAL & 0,00147 & 27,36 & 0,000 \\
OCUP.ELEMENTAL & 0,00035 & 4,11 & 0,000 \\
OCUP.SUPERIOR & 0,00046 & 5,07 & 0,000 \\
CONSTRUCCCION & $-0,00049$ & $-4,08$ & 0,000 \\
INDUSTRIA & $-0,00059$ & $-13,61$ & 0,000 \\
GRANDE & 0,00090 & 22,36 & 0,000 \\
PÚBLICO & 0,00016 & 2,73 & 0,006 \\
EXTERIOR & $-0,00221$ & $-28,54$ & 0,000 \\
REG.EMPRESA & 0,00044 & 8,76 & 0,000 \\
\hline$R^{2}$ & \multicolumn{3}{|c}{0,4946} \\
$\bar{R}^{2}$ & 0,4930 \\
$\hat{\sigma}^{2}$ & & 0,0001 & \\
\hline
\end{tabular}

Fuente: Elaboración propia.

Los resultados de los contrastes espaciales para el modelo pooled se presentan en la Tabla 4.

Tabla 4

Test de independencia espacial del modelo pooled

\begin{tabular}{c|cc}
\hline \multirow{2}{*}{ Test } & \multicolumn{2}{|c}{ IG SAL.ANUAL } \\
\cline { 2 - 3 } & Valor & p-valor \\
\hline$L M_{\lambda}$ & 135,287 & 0,000 \\
$L M_{\lambda}^{*}$ & 102,411 & 0,000 \\
$L M_{\rho}$ & 400,110 & 0,000 \\
$L M_{\rho}^{*}$ & 367,234 & 0,000 \\
\hline
\end{tabular}

Fuente: Elaboración propia.

Los contrastes $L M$, en sus dos versiones, para la ecuación de IG SAL.ANUAL arrojan evidencia de efectos espaciales en forma de retardo endógeno y en el término de error. El modelo pooled puede ser mejorado en su especificación en dos direcciones, una es incluir efectos espaciales en las $X$ 's y la otra es reconocer la pertenencia de las muestras a cada provincia. En la Tabla 5 se presentan las estimaciones del modelo $S L X$ con efectos fijos, tal como se menciona en la Sección 2. 
Tabla 5

Resultados del modelo $S L X$, con efectos fijos

\begin{tabular}{|c|c|c|c|}
\hline \multirow{2}{*}{ Variable } & \multicolumn{3}{|c|}{ IG SAL.ANUAL } \\
\hline & Coef. & t-test & p-valor \\
\hline EDAD 16-29 & $-0,00005$ & $-0,726$ & 0,467 \\
\hline $\mathrm{EDAD}>49$ & 0,00014 & 2,387 & 0,017 \\
\hline EST. PRIMARIOS & 0,00028 & 2,769 & 0,005 \\
\hline EST. SUPERIORES & 0,00044 & 5,649 & 0,000 \\
\hline NAC. NO ESP & $-0,00003$ & $-0,312$ & 0,755 \\
\hline ANTIG $<2$ & 0,00025 & 3,459 & 0,001 \\
\hline ANTIG >10 & 0,00009 & 1,282 & 0,199 \\
\hline TEMPORAL & 0,00043 & 5,233 & 0,000 \\
\hline PARCIAL & 0,00299 & 42,371 & 0,000 \\
\hline OCUP.ELEMENTAL & 0,00028 & 3,489 & 0,000 \\
\hline OCUP.SUPERIOR & 0,00099 & 11,614 & 0,000 \\
\hline CONSTRUCCCION & 0,00498 & 2,529 & 0,011 \\
\hline INDUSTRIA & 0,00071 & 0,693 & 0,488 \\
\hline GRANDE & 0,00184 & 2,114 & 0,034 \\
\hline PÚBLICO & $-0,00064$ & $-0,610$ & 0,541 \\
\hline EXTERIOR & $-0,00139$ & $-0,924$ & 0,355 \\
\hline REG.EMPRESA & 0,00214 & 1,701 & 0,089 \\
\hline W-EDAD 16-29 & $-0,00016$ & $-1,209$ & 0,226 \\
\hline W-EDAD >49 & $-0,00006$ & $-0,418$ & 0,675 \\
\hline W-EST. PRIMARIOS & 0,00030 & 1,431 & 0,152 \\
\hline W-EST. SUPERIORES & $-0,00008$ & $-0,481$ & 0,630 \\
\hline W-NAC. NO ESP & $-0,00018$ & $-0,814$ & 0,415 \\
\hline W-ANTIG $<2$ & 0,00010 & 0,659 & 0,509 \\
\hline W-ANTIG >10 & 0,00026 & 1,661 & 0,096 \\
\hline W-TEMPORAL & $-0,00003$ & $-0,181$ & 0,856 \\
\hline W-PARCIAL & 0,00016 & 1,039 & 0,299 \\
\hline W-OCUP.ELEMENTAL & $-0,00009$ & $-0,498$ & 0,618 \\
\hline W-OCUP.SUPERIOR & 0,00013 & 0,723 & 0,469 \\
\hline W-CONSTRUCCCION & 0,00306 & 0,698 & 0,485 \\
\hline W-INDUSTRIA & 0,00078 & 0,312 & 0,755 \\
\hline W-GRANDE & 0,00241 & 1,287 & 0,198 \\
\hline W-PÚBLICO & 0,00063 & 0,276 & 0,782 \\
\hline W-EXTERIOR & 0,00391 & 1,160 & 0,246 \\
\hline W-REG.EMPRESA & $-0,00333$ & $-1,227$ & 0,219 \\
\hline$R^{2}$ & & 0,8945 & \\
\hline $\bar{R}^{2}$ & & 0,8927 & \\
\hline LR(efectos fijos) & & $5183,63^{\star \star *}$ & \\
\hline
\end{tabular}

Nota: ${ }^{* * \star}$ significancia al 1\%. El test LR contrasta la significancia de los efectos fijos.

Fuente: Elaboración propia.

Los contrastes $L M$ del modelo $S L X$ con efectos fijos son obtenidos a partir de los desarrollos de Debarsy y Ertur (2010). En la Tabla 6 puede observarse que no se rechaza la hipótesis nula de independencia espacial en las versiones simples y robustas para el modelo de IG SAL.ANUAL. Esta evidencia nos indica la idoneidad de estimar un modelo $S L X$ para la concentración anual. 
Tabla 6

Contrastes $L M$ condicionales (efectos fijos)

\begin{tabular}{c|cc}
\hline \multirow{2}{*}{ Test } & \multicolumn{2}{|c}{ IG SAL.ANUAL } \\
\cline { 2 - 3 } & Valor & p-valor \\
\hline$L M_{\lambda}$ & 0,005 & 0,944 \\
$L M_{\lambda}^{*}$ & 0,496 & 0,481 \\
$L M_{\rho}$ & 0,005 & 0,944 \\
$L M_{\rho}^{*}$ & 0,001 & 0,981 \\
\hline
\end{tabular}

Fuente: Elaboración propia.

Una corrección adicional debe ser efectuada. El procedimiento aplicado para obtener las muestras fue realizado de manera independiente entre muestras, sin embargo, debido a que en algunas provincias la encuesta posee un tamaño muestral relativamente pequeño respecto a otras se detectó alta dependencia entre muestras consecutivas. Este hecho genera la necesidad de controlar por parámetros de molestia para evitar ciertos sesgos en los parámetros de interés. Para tal fin, se realizaron un conjunto de modificaciones: (i) se permutaron las muestras conservando la estructura espacial; y (ii) se incluyeron dos parámetros de molestia en el término de error, un coeficiente autorregresivo entre-muestras y un rezago espacial. La introducción de ambos coeficientes en las permutaciones sirve de control para una lectura adecuada de la parte sistemática y se espera que, debido al procedimiento de permutación, ambos sean no significativos.

El procedimiento de remuestreo se basó en 499 permutaciones, a partir de la primera selección muestral. Para cada permutación se estimó un modelo $S L X$ de efectos fijos incluyendo parámetros de molestia en el término de error. Los resultados promedios del procedimiento de permutación se presentan en la Tabla 7. Los resultados de este modelo final, que desde el punto de vista de la modelización espacial se considera como el más idóneo, permiten identificar la significatividad de muchos de los coeficientes del modelo, siendo todos los signos de los coeficientes significativos positivos. Este hecho nos indicaría que se produciría un incremento de la concentración provincial si se incrementase el porcentaje de trabajadores en cada una de las clases consideradas, en detrimento del porcentaje de las clases equivalente. Así, por ejemplo, se deduce un incremento medio de 3 décimas porcentuales en el grado de concentración (expresado éste en porcentaje) por cada punto porcentual que se incremente la contratación a tiempo parcial frente a la de tiempo completo. Este resultado es coherente con la propia definición del contrato, ya que a mayor contratación parcial, mayor desigualdad en el reparto anual de la masa salarial entre los trabajadores. Por cada punto porcentual que se incrementara el porcentaje de trabajadores en el sector de la construcción, la concentración de la provincia se incrementaría en 5 décimas, y en 2 décimas por cada incremento unitario en las 
empresas reguladas por regímenes de empresa o inferior y por cada incremento porcentual unitario de empresas grandes, de más de 200 trabajadores, frente a pequeñas y medianas. En el resto de los casos, las variaciones del grado de concentración quedan reducidas a centésimas porcentuales, siendo las más notorias, la de 9 centésimas de incremento en la concentración por cada punto porcentual que se incremente el porcentaje de trabajadores en ocupaciones superiores o las 4 centésimas de incremento por cada punto porcentual de más en el grupo de los trabajadores con estudios superiores.

Tabla 7

Modelo $S L X$ de efectos fijos Máxima Verosimilitud (499 permutaciones)

\begin{tabular}{l|rrc}
\hline \multicolumn{1}{c|}{ Variable } & \multicolumn{3}{|c}{ IG SAL.ANUAL } \\
\cline { 2 - 4 } & Coef. avg & t-test avg & p-val avg \\
\hline EDAD 16-29 & $-0,00005$ & $-0,739$ & 0,463 \\
EDAD >49 & 0,00014 & 2,395 & 0,017 \\
EST. PRIMARIOS & 0,00028 & 2,799 & 0,005 \\
EST. SUPERIORES & 0,00044 & 5,704 & 0,000 \\
NAC. NO ESP & $-0,00003$ & $-0,317$ & 0,751 \\
ANTIG <2 & 0,00025 & 3,496 & 0,000 \\
ANTIG >10 & 0,00009 & 1,298 & 0,195 \\
TEMPORAL & 0,00043 & 5,279 & 0,000 \\
PARCIAL & 0,00299 & 42,738 & 0,000 \\
OCUP.ELEMENTAL & 0,00028 & 3,529 & 0,000 \\
OCUP.SUPERIOR & 0,00099 & 11,717 & 0,000 \\
CONSTRUCCCION & 0,00500 & 2,557 & 0,011 \\
INDUSTRIA & 0,00071 & 0,701 & 0,485 \\
GRANDE & 0,00184 & 2,128 & 0,034 \\
PÚBLICO & $-0,00064$ & $-0,616$ & 0,539 \\
EXTERIOR & $-0,00140$ & $-0,933$ & 0,352 \\
REG.EMPRESA & 0,00213 & 1,712 & 0,088 \\
\hline W-EDAD 16-29 & $-0,00017$ & $-1,228$ & 0,221 \\
W-EDAD >49 & $-0,00005$ & $-0,415$ & 0,679 \\
W-EST. PRIMARIOS & 0,00030 & 1,427 & 0,155 \\
W-EST. SUPERIORES & $-0,00008$ & $-0,489$ & 0,626 \\
W-NAC. NO ESP & $-0,00018$ & $-0,804$ & 0,423 \\
W-ANTIG <2 & 0,00010 & 0,658 & 0,512 \\
W-ANTIG >10 & 0,00026 & 1,677 & 0,094 \\
W-TEMPORAL & $-0,00003$ & $-0,187$ & 0,851 \\
W-PARCIAL & 0,00016 & 1,054 & 0,293 \\
W-OCUP.ELEMENTAL & $-0,00009$ & $-0,502$ & 0,617 \\
W-OCUP.SUPERIOR & 0,00013 & 0,735 & 0,464 \\
W-CONSTRUCCCION & 0,00311 & 0,713 & 0,477 \\
W-INDUSTRIA & 0,00070 & 0,281 & 0,779 \\
W-GRANDE & 0,00243 & 1,305 & 0,193 \\
W-PÚBLICO & 0,00058 & 0,257 & 0,797 \\
W-EXTERIOR & 0,00386 & 1,150 & 0,251 \\
W-REG.EMPRESA & $-0,00333$ & $-1,233$ & 0,219 \\
\hline AutOC. Espac. en error & 0,01313 & 0,626 & 0,532 \\
\hline AutOC. entre-muestras & $-0,722$ & 0,399 \\
\hline & & &
\end{tabular}

Fuente: Elaboración propia. 
Por último, resulta interesante resaltar el hecho de que la correlación espacial detectada a priori y en los residuos del modelo simple estimado por $\mathrm{MCO}$ a través del contraste del estadístico I de Moran era espuria, en tanto en cuanto no se detecta en las variables independientes espacialmente retardadas de la Tabla 7 (ya que ninguno de los coeficientes es significativo). Es decir, si bien la presencia de dicha correlación espacial era contrastada en los errores del modelo lineal simple, el planteamiento del modelo $S L X$ de efectos fijos con parámetros de molestia ha sido capaz de controlar dicha correlación, dando como resultado unos coeficientes en la Tabla 7 más creíbles que los de la Tabla 3.

En este sentido, se observa que no sólo la estimación de los coeficientes es diferente, sino incluso algunos de sus signos, como es el caso de las variables relacionadas con el porcentaje de trabajadores mayores de 49 años, con estudios superiores, con contrato temporal, o empleados en el sector de la construcción.

\section{CONCLUSIONES}

En este trabajo se ha llevado a cabo un análisis de los determinantes de la concentración salarial en España, con el fin de conocer aquellas estructuras de población trabajadora que más influyen en la cada vez mayor desigualdad salarial presente en el país. El estudio ha sido llevado a cabo desde una perspectiva espacial, debido a la constatación inicial de no poder asumir la independencia espacial de las observaciones, ya que los valores de concentración provincial presentan un determinado patrón espacial.

Tras la especificación de los modelos econométricos espaciales utilizados, se concluye la idoneidad del modelo $S L X$ de efectos fijos, que incluye parámetros de dependencia entre-muestras, para la modelización del grado de concentración salarial medido en términos del salario anual. Los efectos espaciales detectados en el modelo simple son corregidos mediante la inclusión de efectos espaciales en las variables explicativas y en el término error. Ambas inclusiones no muestran efectos significativos y pueden considerarse como variables de control que evitan el sesgo de variables espaciales omitidas a cada una de ellas.

De los resultados obtenidos en este trabajo, se podría afirmar que, si el objetivo político fuera reducir el grado de desigualdad salarial anual a escala provincial, se debería fomentar la implantación de pequeñas y medianas empresas, en detrimento de las grandes, del sector servicios frente al de la construcción, así como incrementar las empresas reguladas por convenios colectivos de ámbito provincial o sectorial y la contratación de trabajadores con estudios profesionales frente a los superiores.

No obstante, dada la estructura porcentual de trabajadores reflejada en esta encuesta, parece claro que un reparto más equitativo de la masa salarial debe venir fundamentalmente de la mano de un mejor reparto de las horas trabajadas, ya que en España las jornadas parciales son muchas veces voluntarias pero tam- 
bién en muchos casos forzadas. Es decir, salvo ese porcentaje de trabajadores que opta por trabajar menos horas semanales, el resto debería poder llegar a repartir las horas de trabajo disponibles si se desea reducir el grado de concentración salarial entre trabajadores. Obsérvese también la particularidad de que la encuesta fue llevada a cabo en 2010, cuando todavía los porcentajes de trabajadores en el sector de la construcción eran demasiado altos para el país. Esto puede implicar la conclusión de que la mayor presencia de trabajadores en el sector incrementa la desigualdad. Así mismo, parece deducirse que sólo de la mano de un menor impacto de la formación y responsabilidad del trabajador en su salario podría verse reducido dicho grado de concentración.

\section{REFERENCIAS BIBLIOGRÁFICAS}

ANSELIN, L.; LE GALLO, J. y JAYET, H. (2006). "Spatial panel econometrics". En Matyas, $\mathrm{L}$ y Sevestre, $\mathrm{P}$ (eds): The econometrics of panel data, fundamentals and recent developments in theory and practice, 3rd edn,(pp. 901-969). Dordrecht: Kluwer.

BASULTO, J. y BUSTO, J.J. (2010). "Gini's Concentration Ratio". Electronic Journal for History of Probability and Statistics, 6: pp. 1-42.

CASTELLS-QUINTANA, D. y ROYUELA, V. (2014). "Agglomeration, inequality and economic growth". The Annals of Regional Science, 52: pp. 343-366.

CASTELLS-QUINTANA, D.; RAMOS, R. y ROYUELA, V. (2015). "Income inequality in European regions: recent trends and determinants". Review of Regional Research, 35: pp. 123-146.

CNAE (2009). "Clasificación Nacional de Actividades Económicas". Disponible en: http://www.ine.es/jaxi/menu.do?type=pcaxis\&path=\%2Ft40\%2Fclasrev\%2F\&file=inebase\&L=1. [Último acceso: 10 octubre 2012].

CNO (2011). "Clasificación Nacional de Ocupaciones". Disponible en: http://www.ine.es/ jaxi/ menu.do?type=pcaxis \&path=\%2Ft40\%2Fcno11\%2F\&file=inebase $\& \mathrm{~L}=1$. [Último acceso: 8 octubre 2012].

DEBARSY, N. y ERTUR, C. (2010). "Testing for spatial autocorrelation in a fixed effects panel data model". Regional Science and Urban Economy, 40: pp. 453-470.

EES (2012a). "Microdatos de la Encuesta de Estructura Salarial, 2010". Instituto Nacional de Estadística. Disponible en: http://www.ine.es/dyngs/INEbase/ es/operacion.htm?c=Estadistica_C\&cid $=1254736176912 \&$ menu=resultados\&secc $=1$ 254736195110\&idp=1254735976596 [Último acceso: 8 octubre 2012].

EES (2012b). "Encuesta de Estructura Salarial" (EES). Metodología. Instituto Nacional de Estadística. Disponible en: http://www.ine.es/metodologia/t22/t223013310.pdf. [Último acceso: 8 octubre 2012].

ELHORST, J.P. (2010). "Spatial panel data models". En Fischer MM y Getis A (eds): Handbook of applied spatial analysis (pp 377-407). Berlin: Springer.

ELHORST, J.P. (2014). Spatial econometrics. From cross-sectional data to spatial panels. Heidelberger: Springer. 
EUROSTAT (2012). "Gini coefficient", Source SILC. Income and Living Conditions Statistics. Disponible en: http://appsso.eurostat.ec.europa.eu/nui/show.do?dataset= ilc_di12\&lang=en. [Último acceso: 20 noviembre 2012].

FMI (2012). Income Inequality and Fiscal Policy, International Monetary Fund Staff Discussion Note. SDN/12/08. June 28, 2012.

GEARY, R. (1954). "The contiguity ratio and statistical mapping". The Incorporated Statistician, 5(3): pp. 115-127.

GILENS, M. (2012). Affluence and Influence. Economic Inequality and Political Power in America. New Jersey: Princeton University Press and The Russell Sage Foundation.

GINI, C. (1912). Variabilità e Mutabilità: contributo allo Studio delle distribuzioni e delle relazioni statistiche. Rome: Università de Cagliari.

GINI. C. (1914). "Sulla misura della concentraciones e della variabilità dei caratteri". Atti del R. Istituto Veneto di Science, Lettere ed Arti 73: pp. 1203-48.

GIORGI, G.M. (2005). "Gini's scientific work: an evergreen". Metro-International Journal of Statistics LXIII(3): 299-315.

HALLECK VEGA, H. y ELHORST, J.P. (2015). "The SLX model". Journal of Regional Science, 3(55): pp. 339-363.

HASSINE. N.B. (2015). "Economic Inequality in the Arab Region". World Development, 66: pp. 532-556.

HATCH, M. y RIGBY, E. (2015). "States can fight growing economic inequality through lowering taxes on the poor, and stricter labor market policies". USApp - American Politics and Policy Blog (27 Jan 2015) Blog Entry. Disponible en: http://blogs.lse.ac.uk/usappblog/ [Último acceso: 10 febrero 2015].

HAUSMAN, J.A. (1978). "Specification tests in Econometrics". Econometrica, 46(6): pp. 1251-1271.

HSIAO, C. (2003). Analysis of panel data. 2nd Edition. New York: Cambridge University Press.

KRISTAL, T. y COHEN, Y. (2015). "Labor Market Institutions and Technology: The Causes of Rising Wage Inequality in U.S. Industries, 1968-2012" (January 14, 2015). Disponible en SSRN: http://ssrn.com/abstract=2549605 or http://dx.doi.org/10.2139/ ssrn.2549605 [Último acceso: 10 febrero 2015].

LARRAZ, B. (2015). "Decomposing the Gini Inequality Index: An Expanded Solution". Sociological Methods\&Research, 44(3): pp. 508-533.

LESAGE, J.P. y PACE, R. (2009). Introduction to Spatial Econometrics. Boca Raton: CRC Press/Taylor \& Francis.

MATÉ SÁNCHEZ-VAL, M.L., GARCÍA PÉREZ DE LEMA, D. y LÓPEZ HERNÁNDEZ, F. (2009). "La influencia de los efectos espaciales en el crecimiento de la productividad de la PYME". Estudios de Economía Aplicada, 27(1): pp. 1-24.

MILANOVIC, B. (2012). Los que tienen y los que no tienen. Una breve y singular historia de la desigualdad global. Madrid: Alianza Editorial.

MORAN, P. (1948). "The interpretation of statistical maps". Journal of the Royal Statisical Society B., 10: pp. 243-251.

MORAN, P. (1950). "Notes on continuous stochastic phenomena". Biometrika, 37: pp. 17-23.

OCHANDO, C. (2010). "La distribución de la renta en España en el período de crecimiento económico: 1998-2005". Estudios de Economía Aplicada, 28-3: pp.1-22. 
PABAYO, R., KAAWACHI, I. y GILMAN, S.E. (2015). "US State-level income inequality and risks of heart attack and coronary risk behaviors: longitudinal findings". International Journal of Public Health, 60(5): pp. 573-588.

PALACIOS, F., GARCÍA, R.M. y LLORCA, C.M. (2014). "The Impact of the Crisis on the Polarization of Spanish Wages". Estudios de Economía Aplicada, 32(1): pp. 335-348.

PARK, C.Y. y MERCADO, R.V.Jr, (2015). "Financial inclusion, poverty and income inequality in developing Asia". ADB Economics Working Paper, 426.

PICKETT, K. y WILKINSON, R.G. (2015). "Income inequality and health: A causal review". Social Science \& Medicine, 128: pp. 316-326.

PIKETTY, T. (2013). El capital en el siglo XXI. Ciudad de México. Fondo de cultura económica.

SNIJDERS, T.A. y BOSKER, R.J. (1999). Multilevel analysis: an introduction to basic and advanced multilevel modeling. Thousand Oaks: Sage Publications.

STIGLITZ, J.E. (2012).The Price of Inequality: How Today's Divided Society Endangers Our Future. New York: W.W. Norton \& Company.

VICENTE-PERDIZ, J., RUBIO, M.T., y DECIMAVILLA, E. (2010). "World's Growth and Inequalities". Estudios de Economía Aplicada, 28(2), pp. 1-14.

ZMERLI, S. y CASTILLO, J.C. (2015). "Income inequality, distributive fairness and political trust in Latin America". Social Science Research, 52: pp.179-192. 
